\title{
Fighting osteoporosis black and blue
}

Researchers in Japan have identified delphinidin as the major anthocyanidin compound in blue berries (bilberries, blackcurrants) that can inhibit osteoclastogenesis and thereby the development of osteoporosis in mice.

Shumpei Niida, corresponding author of the work (published in PLoS ONE), says his research complements an "epidemiological study that demonstrates that anthocyanin was beneficial for maintenance of bone mineral density".

Niida's team found anthocyanidins had no substantial effect on mouse osteoblast cultures, but pretreatment with delphinidin inhibited receptor activator of NF- $\kappa \mathrm{B}$ ligand (RANKL)-induced osteoclastogenesis of RAW264.7 cells by reducing the expression of $c$-fos, Nfatc 1 and osteoclast marker genes $M m p 9$ and Trap, and interfering with NF- $\kappa \mathrm{B}$ signalling. Importantly, delphinidin treatment was dose-dependent, nontoxic and superior to other anthocyanidins (cyanidin and peonidin) in these effects.
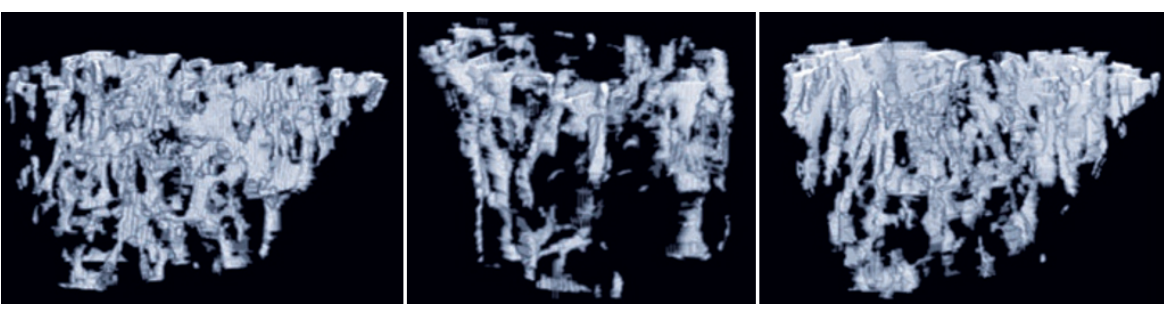

Micro-CT images of mouse tibiae. Control (left panel). RANKL induces bone loss (middle panel), but pretreatment with delphinidin prevents RANKL-induced bone loss (right panel). Images courtesy of S. Niida.

The researchers also administered the compound orally to ovariectomized mice and to mice with RANKL-induced osteoporosis. Femurs were scanned by microcomputed tomography to measure bone density parameters, including trabecular number, thickness, separation and spacing. Delphinidin treatment (figure, right panel) prevented cancellous bone degradation (figure, middle panel) in both mouse models.

In response to Niida's publication, Mone Zaidi, an osteoporosis expert at the Icahn School of Medicine at Mount Sinai (NY, USA) notes the "renewed interest in understanding effects of natural products on bone," but he cautions that for drug development "the challenge is to define the molecular targets for anthocyanins". Until then, dietary supplementation with blue berries might help in the fight against osteoporosis.

Nicholas J. Bernard

Original article Moriwaki, S. et al. Delphinidin, one of the major anthocyanidins, prevents bone loss through the inhibition of excessive osteoclastogenesis in osteoporosis model mice. PLOS ONE doi:10.1371/ journal.pone.0097177 


\section{CORRECTION}

Bone: Fighting osteoporosis black and blue

Nicholas J. Bernard

Nat. Rev. Rheumatol. advance online publication 3 June 2014; doi:10.1038/nrrheum.2014.88

In the original version of this article, the second paragraph incorrectly referred to the original author of the research as 'Sumpei Niida' instead of 'Shumpei Niida'. This error has now been corrected in the HTML and PDF versions of the article. 